\title{
Cortisol: the villain in Metabolic Syndrome?
}

\author{
Sílvia Paredes ${ }^{1}$, Laura Ribeiro ${ }^{2}$ \\ 1- Faculty of Medicine, University of Porto \\ 2- Department of Biochemistry and Center for Medical Education, Faculty of Medicine, University of Porto
}

Study conducted at Department of Biochemistry, Faculty of Medicine, University of Porto, Porto, Portugal

Article submitted: 10/04/13 Accepted for publication: 05/08/13

Correspondence Laura Ribeiro Department of Biochemistry, Faculty of Medicine, 4200-319 Porto, Portugal Phone: 351225513654 Fax: 351225513655 Iribeiro@med.up.pt

http://dx.doi.org/10.1590/1806-9282.60.01.017 Conflict of interest: none

\section{SUMmARY}

Objective: This article reviews the state of the art regarding the association between glucocorticoid actions and both obesity and insulin resistance, two main features of the metabolic syndrome.

Methods: A methodological assessment of the literature on PubMed and SciELO databases was conducted by using the following terms: stress, metabolic syndrome, glucocorticoids, obesity, insulin resistance, hypothalamic-pituitary-adrenal-axis and 11ß-hydroxysteroid dehydrogenase.

Results: Chronic stress, mainly through hypothalamic-pituitary-adrenal axis dysregulation, promotes the accumulation of visceral fat. Reciprocally, obesity promotes a systemic low-grade inflammation state, mediated by increased adipokine secretion, which can chronically stimulate and disturb stress system. This vicious cycle, probably initiated by visceral adipose tissue dysfunction, might be the trigger for the development of metabolic syndrome.

Conclusion: Given the strong evidences linking glucocorticoid release, obesity and type 2 diabetes, better understanding of the mechanisms underlying this connection might be useful for prevention and treatment of the metabolic syndrome.

Key words: stress; glucocorticoids; obesity; insulin resistance; metabolic Syndrome; $11 \beta$ Hydroxysteroid; dehydrogenase.

\section{INTRODUCTION}

Stress is defined as a state of threatened homeostasis ${ }^{1}$, and comprises a complex repertoire of physiologic and behavioral responses that aim to restore the challenged body equilibrium ${ }^{2}$. The hypothalamic-pituitary-adrenal (HPA) axis and the central and peripheral components of the autonomic nervous system are responsible for crucial functions of the stress system ${ }^{3}$. Acute activation of stress reaction leads to a cluster of time-limited, behavioral and physical changes that are normally adaptive and aim to improve the chances of individuals to survive $^{3}$. Inadequate, excessive, and/or prolonged reactions to stress may lead to disease ${ }^{4}$. Chronic stress, a prolonged threat to homeostasis by persistent or frequently repeated stressors, is an important aspect of daily life leading to the development of a wide range of diseases and syndromes ${ }^{3}$. In fact, long exposure to stress can disrupt the pathways involved in metabolism, growth, reproduction, immunity, personality and behavior development ${ }^{1}$.
The metabolic syndrome (MS) can be described as a cluster of metabolic abnormalities, in which fat accumulation appears to play a central role and has a thigh relationship with type 2 diabetes ${ }^{5}$. Obesity has become recognized as one of the major health problems nowadays with a strong and deleterious impact on health status and health-care $\operatorname{costs}^{6}$. In order to cope with periods of starvation, humans were genetically programmed to accumulate high amounts of energy. However, modern lifestyles offer open access to food and promote sedentary habits, leading to a progressive cycle of overeating and weight gain. The prevalence of type 2 diabetes mellitus, an important cause of mortality and morbidity worldwide, is expected to worsen in the next decades $^{7}$. The hypothesis that an adverse psychosocial environment contributes to the development of obesity and type 2 diabetes ${ }^{8}$ has been raised. In fact, both intense stress reactivity and an abnormal recovery predict MS development ${ }^{9}$. A long exposure to psychological stress may impair the organism capacity to maintain biological balance and thus 
disrupt homeostasis ${ }^{10}$, causing adverse metabolic effects. Several studies have shown a significant association between glucocorticoids (GC), visceral fat, type 2 diabetes and $\mathrm{MS}^{2,11}$.

\section{Methods}

In this article we review recent data suggesting that GC excess is implicated in the development of the metabolic complications that characterize central obesity and MS. A methodological assessment of the literature on PubMed and SciELO databases was conducted without setting limits for year publication but selecting both English and Portuguese papers with full text availability. The following terms were used: stress, metabolic syndrome, glucocorticoids, obesity, insulin resistance, hypothalamic-pituitary-adrenal-axis and $11 \beta$-hydroxysteroid dehydrogenase.

\section{Stress AND MEdiators OF STRESS RESPONSE - HPA AXIS}

The HPA axis is important in maintaining a dynamic equilibrium or homeostasis in a constantly changing environment $^{3}$. The paraventricular nucleus of the hypothalamus contains neuroendocrine neurons that synthesize and secrete vasopressin (AVP) and corticotropin-releasing hormone (CRH). CRH and AVP are released from neurosecretory nerve terminals at the median eminence. $\mathrm{CRH}$ is transported to the anterior pituitary through the portal blood vessel system of the hypophyseal stalk and AVP is transported by axonal transport to the posterior pituitary. There, CRH and AVP act synergistically to stimulate the secretion of stored adrenocorticotropic hormone (ACTH) from corticotrope cells. After release into the bloodstream, ACTH reaches the adrenal gland where it rapidly stimulates the biosynthesis of $\mathrm{GC}^{3,12}$. GC, in turn, inhibit $\mathrm{CRH}$ at the hypothalamic level, and interfere with ACTH-secre- tion in the anterior pituitary, thereby establishing a regulatory feedback loop ${ }^{13}$. The activity of this endocrine system is characterized by a robust circadian rhythm with cortisol levels peaking in the early morning hours around the time of awakening and being lowest around midnight ${ }^{14}$. Diurnal variations are modulated by changes in lighting, feeding schedules, and physical activity, and are disrupted in face of a stressor ${ }^{3}$. Inflammatory mediators can also be secreted in response to different stressors and can activate the HPA axis ${ }^{15}$. For instance, tumor necrosis factor- $\alpha$ (TNF $\alpha$ ), interleukin-1 (IL-1) and interleukin-6 (IL-6) are mainly present in states of chronic inflammatory stress and can activate HPA axis $^{15}$. GC are present in the systemic circulation mainly bound to corticosteroid-binding globulin (CBG) (approximately 90\%), and also to albumin (4$5 \%$ ). Only $5-6 \%$ of the total circulating GC remain in an unbound state and, thus, biologically active ${ }^{14}$. CBG may have, either a buffer function blunting elevations of free cortisol during a secretory peak or a reservoir role maintaining cortisol pool during times of reduced secretion ${ }^{16}$. As mentioned above, GC exert an inhibitory feedback action in the stress response system, a fundamental action in limiting the duration of total GC tissue exposure, thus minimizing their catabolic, adipogenic, antireproductive, and immunosuppressive effects ${ }^{3}$. GC exert their actions by binding to two types of intracellular receptors: the glucocorticoid receptor (GR) which responds to high levels of GC and the mineralocorticoid receptor (MR) responding to low levels of $\mathrm{GC}^{13}$. GC modulate the expression of a widerange of genes in a DNA-dependent and independent manner ${ }^{3,14,17}$. It is of note that numerous genes encoding important proteins that are directly or indirectly implicated in several metabolic pathways are rigorously regulated by GC (Table 1$)^{14}$. 11 $\beta$-Hydroxysteroid Dehydrogenase

TABLE 1 Selected glucocorticoid sensitive genes involved in important metabolic pathways

\begin{tabular}{l|l} 
Upregulated genes - Pathway affected & Downregulated genes - pathway affected \\
\hline Leptin - Energy homeostasis & CRH - HPA axis regulation \\
GLUT4 - Glucose transport & POMC - HPA axix and appetite control \\
Glucose-6-phosphatase - Gluconeogenesis & TNF- $\alpha$ - Proinflammatory response \\
Hepatic PEPCK - Gluconeogenesis & Interleukin-6 - Proinflammatory response \\
Lipoprotein lipase - Lipid metabolism & Interleukin-8 - Proinflammatory response \\
Hormone sensitive lipase - Lipolysis & Adiponectin - Insulin signaling, Atherogenesis \\
VLDL receptor - Lipoprotein metabolism & Prolactin - Reproducion \\
Tyrosine aminotransferase - Amino acid catabolism & Osteocalcin - Bone metabolism \\
Tryptophan oxygenase - Amino acid catabolism & \\
\hline
\end{tabular}

GLUT4: Glucose transporter 4; PEPCK: Phoshpoonolpyruvate carboxylinase; VLDL: Very low density lipoprotein; CRH: Corticotropin hormone; HPA: Hupothalamic-pituitary-adrenal; POMC: Prooplomelanocortin; TNF- $\alpha$ : Tumor necrosis factor- $\alpha$.

Adapted from Kyrou\&Tsigos ${ }^{1}$ 
(11 $\beta$-HSD) catalyzes the interconversion of inactive cortisone to active cortisol, or vice-versa ${ }^{14}$. $11 \beta$-Hydroxysteroid Dehydrogenase type $2(11 \beta-H S D 2)$ predominates in renal tubules and protects the MR from excessive stimulation by cortisol. It has also been identified in colon, salivary glands and placenta ${ }^{18}$. The apparent mineralocorticoid excess syndrome results from defective $11 \beta$-HSD2. Indeed, its deficiency allows an increase of cortisol levels and MR activation inducing sodium retention, hypokalemia and hypertension ${ }^{19}$. 11ß-Hydroxysteroid Dehydrogenase type 1 (11ß-HSD1), which is mostly expressed in liver, fat, gonadal tissue and central nervous system, is believed to function as a reductase, generating active cortisol at a prereceptor level, thus enhancing the activation of $\mathrm{GC}^{20}$. The co-localization of 11 $\beta$-HSD1 with Hexose-6-phosphate dehydrogenase $(\mathrm{H} 6 \mathrm{PDH})$ has an important role in providing nicotinamide adenine dinucleotide phosphate (NADPH) as cofactor to drive the direction to $11 \beta-H S D 1$ reductase, rather than to dehydrogenase ${ }^{18}$.

\section{Physiological effects of glucocorticoids}

GC are the final mediators of HPA axis activation, playing a key role in modulating immunological and inflammatory responses, energy metabolism and cardiovascular homeostasis and general responses to stress ${ }^{18}$. In order to face threats imposed by stressors, several responses are activated towards the restoration of homeostasis. Behavioral adaptations include increased arousal, euphoria and cognition, enhanced analgesia, and sleep inhibition. Physical adaptations include increases in cardiovascular tone, respiratory rate, and metabolism. The activation of HPA axis promotes a redirection of energy, in order to deliver oxygen and nutrients to organs and tissues involved in the adaptation functional system. In the meantime, other non-emergent functions, such as digestion, reproduction, growth, and overall immunity, are temporarily suppressed $^{3,12}$. All these acute effects increase the capacity for generation of energy over a limited period of time improving the ability to 'fight or flight ${ }^{20}$. GC induce lipolysis, even though they favor both abdominal and dorsocervical fat accumulation ${ }^{17}$. Given that GC promote delivery of fuel to skeletal muscles, the increase in triglycerides (TAG) stores in adipose tissue in clinical conditions with GC excess appears paradoxical. This paradox probably reflects the combination of elevated GC levels concomitantly with elevated insulin levels in individuals who are able to ingest unrestricted energy without consuming it - for instance, through physical exercise. In these circumstances, fatty acid esterification predominates over lipolysis and, combined with stimulation of pre-adipocyte differentiation, promotes fat accumulation $^{20,21}$, a mechanism that is most probably common to what happens under chronic stress.

GC increase hepatic gluconeogenesis and decrease glucose uptake and insulin sensitivity, thus favoring hyperglycemia. In order to provide amino acids as an additional substrate for oxidative pathways, GC cause protein degradation at multiple tissues such as muscle, bone and skin. GC also antagonize the anabolic actions of growth and thyroid hormones, insulin, and sex steroids on their target tissues ${ }^{3,12}$. Globally, stress response is responsible for a shift of normal metabolism, favoring a catabolic state which returns to normal after stress removal. Chronic exposure to stress, however, can be potentially damaging, as long exposure to GC can dysregulate multiple metabolic pathways leading to a progressively increase in visceral adiposity, hyperglycemia, dyslipidemia, hypertension and insulin resistance ${ }^{3,12}$. Elevated circulating GC levels also result in myopathy, osteoporosis, osteonecrosis, mental disturbances, increased susceptibility to infections and infertility ${ }^{12,20}$. GC actions in the central nervous system are complex ${ }^{12}$. GC affect the capacity to apprehend sensations and establish suitable reactions to stimuli $^{22}$, modulate behavior and humor and interfere with memory retention ${ }^{4}$. Short-term changes in immunological function may be valuable, preventing the damage caused by sustained exposure to various cytokines ${ }^{3}$.

\section{Metabolic Syndrome}

The MS is a cluster of metabolic abnormalities that increase the risk for type 2 diabetes mellitus and cardiovascular disease. It can be defined as a state of disturbed metabolic homeostasis characterized by the combination of central obesity, insulin resistance, dyslipidemia and hypertension ${ }^{17}$. The worldwide incidence of both obesity and MS has been significantly rising in the last decades, threatening to become the new epidemic of this century. In fact, currently, MS affects $1 / 4$ of the adult Portuguese population ${ }^{23}$. It is noteworthy that these clinical conditions often show a relationship with indices of stress ${ }^{24}$. Although obesity per se is not a required feature for the diagnosis of MS, several evidences suggest that both visceral obesity and insulin resistance have a key role in the pathogenic mechanisms underlying this syndrom $e^{25}$. The distribution of fat seems to be a powerful predictor of cardiometabolic disease ${ }^{26}$ and many prospective studies have shown that central obesity is more often correlated to the features of $\mathrm{MS}^{25}$. This pathophysiologic relationship is consistent with the emphasis on waist measurement for MS criteria rather than body mass index (BMI), 
as an indicator of obesity-related cardiovascular risk ${ }^{25}$. In fact, even in normal-weight subjects, increased abdominal circumference is associated with increased risk for cardiovascular disease ${ }^{27}$. The strong literature evidences highlighted in the next sections reinforce our opinion in that GC, released in response to chronic stress, induce an accumulation of visceral fat that, when some conditions are met (such as a lifestyle characterized by excessive energy intake, low physical activity and low quality food), will trigger the development of MS.

\section{Clinical associations between hypercorti- SOLISM AND METABOLIC SYNDROME}

The similarities between the clinical features of Cushing syndrome and those of MS raised the hypothesis that MS is associated with GC excess ${ }^{9,14}$. Cortisol excess has been implicated in the development of diabetes and obesity, highlighting the role of psychological stress on both conditions (Figure 1$)^{17}$. Indeed, human studies have documented that abdominal obesity and its metabolic comorbidities are significantly correlated with stress-related conditions such as adverse life events, psychological disturbances, and psychosocial problems ${ }^{2}$. In fact, the individual inability to cope with long-term environmental adverse stressful events has been related to HPA axis hyperactivation in obesity, particularly the visceral phenotype ${ }^{9}$. Chronic work stress also seems to predict general and central obesity during midlife ${ }^{1}$. Prolonged and nonremitting stress may result in chronic hyperactivation of the HPA axis with resulting sustained GC release $e^{28}$, which can progressively cause visceral fat accumulation and insulin resistance. In line with this, patients with MS seem to have HPA axis hyperactivity and a functional hypercortisolism ${ }^{14,29}$. On the contrary, in obese individuals, the circulating GC levels have been reported as normal or even low ${ }^{30,31}$. Several hypotheses can explain an abnormal secretion of cortisol in obesity. Firstly, the presence of a primary neuroendocrine abnormality can cause an irregular central drive to $\mathrm{CRH}, \mathrm{ACTH}$ and cortisol. Second, an altered peripheral metabolism of cortisol due to dysregulation of $11 \beta$-HSD1 can also explain normal or low cortisol concentrations in obesity ${ }^{32}$. On the other hand, it has been suggested that cortisol production rate may increase as the amount of visceral fat enlarges ${ }^{33}$, however, as said before, in most cases cortisol plasma concentrations are normal or even low. This might be partly explained by enhanced metabolic clearance of cortisol $^{17}$, due to a combination of enhanced $5 \alpha$-redutase activity and impaired regeneration of cortisol from cortisone by $11 \beta$-HSD 1 in the liver, which results in the increase of

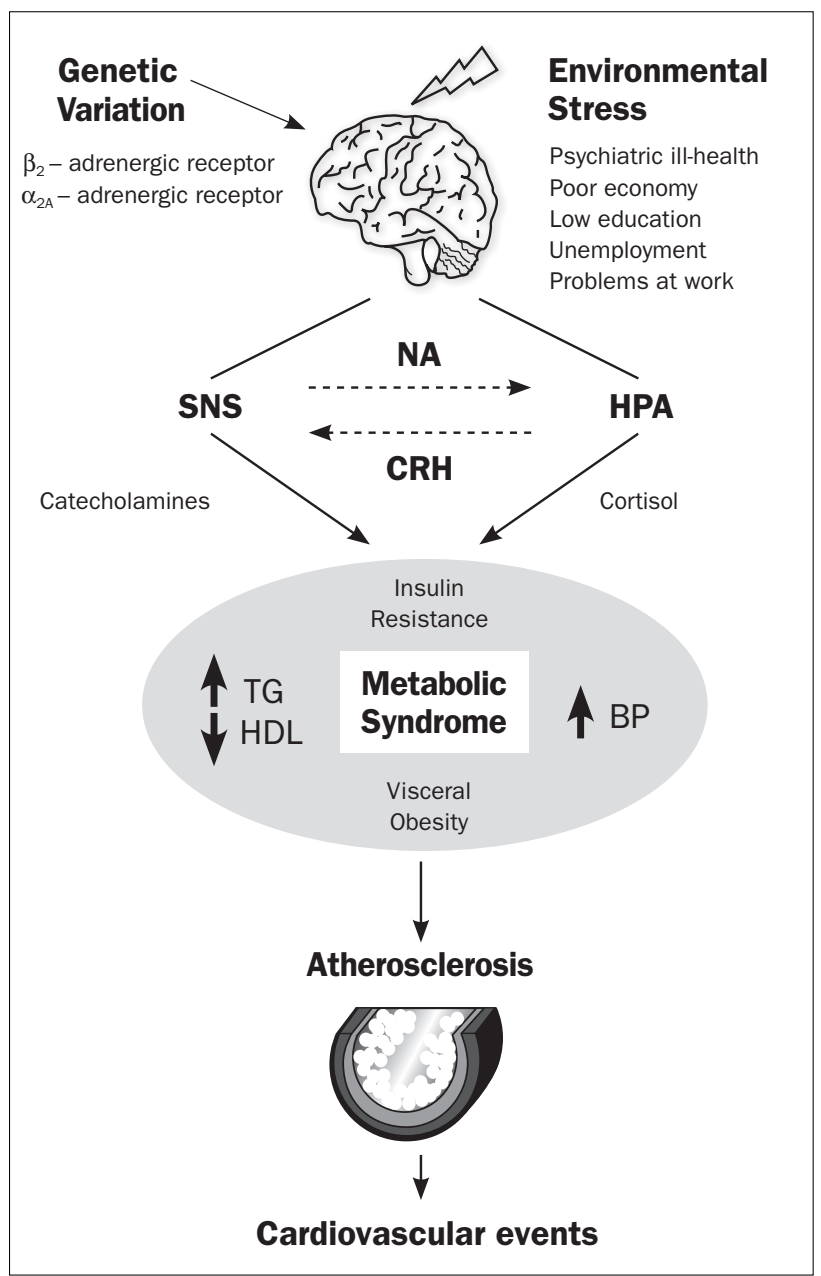

FIGURE 1 The stress response leads to activation of two major neurohumoral systems, the hypothalamic-pituitary-adrenal (HPA) axis and the sympathetic-nervous-system (SNS) which, through the release of cortisol and catecholamines, respectively, exert crucial roles upon energy metabolism, ultimately leading to the development of features of the metabolic syndrome. TG - Triglycerides; HDL - High-density lipoprotein; BP - Blood pressure; NA-noradrenaline; $\mathrm{CRH}$-corticotropin-releasing hormone (Adapted from Rosmond $\mathrm{R}^{21}$ ).

cortisol urinary metabolites excretion ${ }^{32}$. In accordance with this, it has been shown that patients with obesity and MS show increased urinary excretion of free cortisol and its metabolites ${ }^{17}$. Interestingly, the excretion of urinary free cortisol correlates with anthropometric parameters of visceral fat distribution ${ }^{32}$. Overall, there appears to be hyperactivity of the HPA axis in response to stress in patients with visceral obesity ${ }^{2,17}$, which is in accordance with results that show a higher release of cortisol after stimulation with ACTH and greater ACTH release after $\mathrm{CRH}$ infusion ${ }^{30,33}$. Moreover, chronically active HPA axis has an inadequate suppression by dexamethasone ${ }^{9}$. Sev- 
eral studies have shown that in obesity different stimulus such as some neuropeptides, psychological stress and mixed meal tests induce hyperactivation of HPA axis. Individuals that show elevated levels of cortisol in response to perceived stress show a higher association with central fat mass and signs of $\mathrm{MS}^{9}$. Animal studies demonstrated that cynomolgus monkeys subjected to high stress levels comparably to controls secreted higher amounts of GC and were less sensitive to their negative feedback ${ }^{28}$. Another study reported that these animals undergoing physical and psychological stress presented higher basal cortisol levels and increased cortisol release after ACTH stimulation, which was associated with greater visceral fat accumulation ${ }^{24}$. In adipose tissue, lipids are stored as TAG. GC increase lipolysis in adipocytes, as a result of increased transcription and expression of the lypolytic proteins adipose triglyceride lipase and hormone-sensitive lipase, increasing the amount of fatty acids in circulation, which, in turn, contribute to triglyceride accumulation in other tissues ${ }^{34}$. In the liver, GC increase the expression of fatty acid synthase increasing lipid production, thus favoring hepatic steatosis. GC also promote the secretion of lipoproteins ${ }^{14}$. TAG in circulation, as components of both very low-density lipoproteins and chylomicrons, when hydrolyzed, release fatty acids that can be taken up by the surrounding tissues for use or storage, mainly in liver, muscle and central adipocytes ${ }^{34}$. GC promote the differentiation of adipose stromal cells to mature adipocytes, increasing visceral fat accumulation ${ }^{34}$ and redistributing adipose tissue from peripheral to central depots, and increasing the size and number of fat cells $\mathrm{s}^{17,35}$.

Adenosine monophosphate activated protein kinase (AMPK) is a sensor of cellular energy status and is activated in response to a decrease of this state. When activated, AMPK stimulates appetite in the hypothalamus and switches anabolic into catabolic pathways, such as glycolysis and fatty acid oxidation. GC inhibit the AMPK system thus contributing to central fat deposition ${ }^{36}$. GC increase caloric and dietary fat intake ${ }^{34}$ and suppress thermogenesis 9

Growing evidence suggests that there is a relationship between type 2 diabetes and chronic stress disorders ${ }^{1}$. In fact, circulating cortisol concentrations are higher in people with glucose intolerance and type 2 diabetes $^{37}$. GC raise blood glucose levels through several mechanisms ${ }^{38}$. GC impair the insulin-dependent glucose uptake in peripheral tissues, enhance glucose production in the liver and inhibit insulin secretion from pancreatic $\beta$-cells. Thus, cortisol excess can be correlated with diabetes mellitus in clinical settings ${ }^{38}$. Insulin stimulates translocation of the GLUT4 glucose transporters from intracellular compart- ments to plasma membrane, increasing the rate of glucose utilization, however this action is inhibited by high levels of $\mathrm{GC}^{14}$. In insulin sensitive tissues, such as liver and skeletal muscle, GC also impair pathways involved in insulin receptor activation ${ }^{2,38}$. GC promote gluconeogenesis by stimulating the expression of phosphoenolpyruvate carboxykinase (PEPCK) and glucose-6-phosphatase (G6Pase), the rate-limiting enzymes of this pathway, resulting in increased hepatic glucose output and hyperglycemia ${ }^{14,38}$. GC decrease GLUT2 expression in pancreatic $\beta$-cells and impair calcium disposal on insulin secretory process, thus affecting its secretion ${ }^{14}$.

Obesity seems to be implicated in the development of insulin resistance $e^{25,27}$. In fact, pathophysiological accumulation of lipids in the liver has been identified as an independent risk factor for insulin resistance and $\mathrm{MS}^{13}$. Free fatty acids inhibit insulin secretion by pancreas and decrease glucose uptake ${ }^{39}$. GC release increases lipolysis generating free fatty acids into the circulation, which in turn impair insulin signaling pathways downstream of its receptor, thus promoting insulin resistance ${ }^{27,39}$. In humans, the administration of dexamethasone decreases glucose oxidation, which can be due to an increase of free fatty acids plasmatic levels ${ }^{40}$. Thus, insulin resistance can result from an excess of visceral fat $t^{27,41}$. This is in accordance with our view, that visceral fat might be the main trigger for the development of MS. On the other hand, insulin resistance impairs lypogenesis, thus increasing the plasmatic levels of free fatty acids, creating a vicious circle $^{39}$. Adipokines play an important role on fat accumulation and insulin resistance ${ }^{27,39}$. Adiponectin modulates a number of metabolic processes, including glucose homeostasis and fatty acid oxidation. It promotes insulin sensitivity and is negatively regulated by $\mathrm{GC}^{17}$. Hypoadiponectinemia is an independent risk factor for developing MS and type 2 diabetes mellitus ${ }^{42}$. Leptin plays a key role in regulating both energy intake and metabolism, and its circulating levels are directly proportional to body fat ${ }^{39}$. Activation of hypothalamic leptin receptors suppresses appetite, induces satiety and increases energy expenditure ${ }^{43}$. High sustained concentrations of leptin released from adipose tissue result in desensitization of leptin receptor leading to leptin resistance ${ }^{39,44}$. Unusually high circulating leptin levels and low adiponectin levels are generally exhibited by patients with obesity, insulin resistance and $\mathrm{MS}^{27}$. Visfatin is a recently discovered adipokine with insulin-mimetic properties ${ }^{25}$. ACTH reduces the expression of both visfatin and adiponectin, thus promoting a temporary state of insulin resistance. Neuropeptide Y (NPY) release in response to HPA axis ac- 
tivation seems to promote abdominal fat storage. GC stimulates NPY hypothalamic secretion and up-regulate the NPY Y2 receptor in visceral fat. NPY increases food intake and storage of energy as fat and NPY Y2 receptor activation stimulate fat angiogenesis, proliferation and differentiation of new adipocytes ${ }^{44}$.

GR signaling plays a significant role in metabolic regulation, and defects in this signaling pathway have been implicated in the development of several phenotypes of MS. In fact, insulin resistance is associated with increased GR expression in skeletal muscle ${ }^{38}$. Several polymorphisms related to the HPA axis have been associated with HPA abnormal function and development of $\mathrm{MS}^{21,44}$. Chronic stress disorders are commonly associated with behavioral changes which lead to weight gain and metabolic abnormalities. Chronic stress seems to promote unhealthy behaviors such as a sedentary lifestyle, alcohol consumption, smoking and overeating ${ }^{1}$. Stress system affects the hypothalamic appetite-satiety centers therefore disturbing food intake $e^{3}$. The relationship between stress and food ingestion has been extensively investigated but has led to conflicting results. Increasing food intake during stress diminishes HPA axis response to stressors ${ }^{45}$, which might explain why various individuals overeat when exposed to stress. Food ingestion to relieve anxiety is a harmful coping strategy and can lead to undesirable weight gain and obesity ${ }^{46}$. On the other hand, acute stress may be accompanied by a decrease on food consumption. Indeed, acute elevations of $\mathrm{CRH}$ can cause anorexia and stimulate energy expenditure. As mentioned before, NPY increases food intake and storage of energy as fat, and acute stress inhibits NPY release ${ }^{3}$. On the contrary, chronic stressful situations have the opposite effect and people experiencing a high stress reactivity tend to have a greater caloric intake, preferably dense calories ${ }^{46}$. In fact, long-term exposure to circulating GC seems to enhance consumption of high fat and highly palatable foods ${ }^{45}$. GC also decrease energy expenditure ${ }^{3}$ and diminish signs of satiety ${ }^{46}$. A high caloric intake is a mechanism that seems natural in response to fasting. However, this is not the case of psychological stress conditions, in which food is used rather to relief anxiety than to overcome fasting ${ }^{34,45}$.

\section{1ß-HSD1 ROLE}

Tissue-specific dysregulation of GC metabolism seems to be involved in the complex pathophysiology of obesity and the $\mathrm{MS}^{35}$. In fact, 11ß-HSD1 expression positively correlates with obesity and insulin resistance ${ }^{35}$. As mentioned before, circulating GC concentrations are occasionally abnormal in human obesity, however locally enhanced responsiveness to GC has also been implicated in MS development ${ }^{1,17,19,38}$.

Obese individuals have a tissue-specific $11 \beta$-HSD1 dysregulation ${ }^{32,37}$ in which $11 \beta$-HSD1 activity is found selectively increased in visceral fat depots and decreased in liv$\mathrm{er}^{20,29}$. The relationship between the $11 \beta-H S D 1$ function and metabolic disorders has been well established by studies using genetically modified rodent models. In fact, up regulation of 11ß-HSD1 expression selectively in adipose tissue leads to a model of MS in mice ${ }^{19,38}$. Mice with a similar degree of $11 \beta-H S D 1$ over expression in the liver show an attenuated MS profile without visceral obesity ${ }^{29}$. On the contrary, knockout mice lacking $11 \beta$-HSD1 exhibit protection for MS features ${ }^{27,29,37}$. Peroxisome proliferatoractivated receptors (PPAR) are a group of nuclear receptor proteins involved in adipocyte differentiation and fat redistribution to the peripher ${ }^{47}$. 11ß-HSD1 knockout mice show higher expression of PPAR $\gamma$ receptor in all adipose tissue depots ${ }^{38}$.

\section{Obesity as a chronic inflammatory state}

In recent years, it has become clear that obesity is a state of chronic low-grade inflammation ${ }^{27}$. The association between insulin resistance and the other components of MS can be a consequence of their common outcomes as lowgrade inflammation states ${ }^{27,48}$. Adipose tissue releases cytokines that initiate a state of low-grade inflammation resulting in the metabolic, hemodynamic and vascular consequences of this state ${ }^{41,48}$. The uninterrupted release of these pro-inflammatory adipokines is a chronic stimulus for HPA axis activation, creating a vicious cycle, in which hypercortisolemia promotes adipocyte growth and vice versa ${ }^{3}$. The pro-inflammatory cytokines, TNF $\alpha$, IL-1 and IL- 6 act synergistically activating HPA axis and increasing $11 \beta$-HSD1 expression in adipose tissue ${ }^{14,17}$. IL-6 concentrations have a strong correlation with visceral obesity and are associated with insulin resistant $t^{39,41}$. There is a positive association between TNF $\alpha$ concentrations and $\mathrm{BMI}$, and this cytokine seems to be implicated in insulin resistance development $t^{27,33,41}$. Thus, pro-inflammatory cytokines are important players in this process probably starting with the dysfunction of visceral adipose tissue towards the development of other components of MS.

\section{GLUCOCORTICOIDS INHIBITORS AS POTENTIAL THERAPEUTIC TARGETS}

To date, no single agent can ameliorate the underlying causes of $\mathrm{MS}^{27}$. Additional research is needed for novel agents to effectively treat the multiple abnormalities of this syndrome. Antagonizing GC action has been taken 
as an approach to treat some of the MS features ${ }^{14}$, leading to decreases in adiposity, glucose intolerance and insulin resistance, lowering fasting blood glucose and normalizing its postprandial levels ${ }^{20,29,38}$. However, longterm systemic treatment with a GR antagonist may not be a viable option, since it can excessively activate the HPA axis causing adrenal hyperplasia and undesirable increase in cortisol, androgens and mineralocorticoids ${ }^{19,38}$. On the other hand, selective 11ß-HSD1 inhibitors have shown considerable potential for MS treatment. Over the past years, clinical studies have been conducted for several $11 \beta$-HSD1 inhibitors ${ }^{29,38}$. The ability to decrease intracellular cortisol levels in liver and adipose tissue, without altering circulating cortisol concentrations or responses to stress, might be an exciting therapeutic strategy for obesity, type 2 diabetes mellitus and MS treatment. In fact, these drugs have been shown to ameliorate metabolic abnormalities, by improving lipid profile, insulin sensitivity, promoting glucose tolerance and blocking adipogenesis ${ }^{17,38}$. However, there are some concerns regarding the use of these drugs. $11 \beta$-HSD1 inhibition on hippocampus might decrease central feedback inhibition, which may cause HPA axis activation with increased GC release and enhancement of its effects in peripheral tissues. Moreover, non-selective compounds can potently inhibit $11 \beta$-HSD1 causing apparent mineralocorticoid excessive release with sodium retention, hypertension and hypokalemia ${ }^{38}$. Nevertheless, PPAR $\alpha$ and PPAR $\gamma$ agonists are able to down regulate 11ß-HSD1 activity in liver and adipose tissue, respectively ${ }^{27}$, constituting promising approaches. Emerging data suggest that dietary habits have a role on $11 \beta$-HSD 1 modulation. For instance, naturally occurring $11 \beta$-HSD 1 inhibitors include polyphenols, such as flavones and querce$\operatorname{tin}^{29}$. Coffee has been reported to have anti-diabetic effects due to its ability to impair hepatic gluconeogenesis and inhibit 11 $\beta$-HSD1 function ${ }^{29}$. Dietary trans and saturated fatty acids appear to be involved in the development of MS, through their ability to up regulate $11 \beta$-HSD1, thus, increasing local amplification of GC action in adipose tissue ${ }^{29}$. Sucrose promotes simultaneously a decrease in hepatic $11 \beta$-HSD 1 and an increase in $11 \beta$-HSD1 in adipose tissue. Dietary sucrose increases H6PDH, which, in turn, may enhance 11ß-HSD1 activity and intracellular GC. These observations support the fact that increased activity of $11 \beta-H S D 1$ in response to sucrose ingestion is able to cause obesity ${ }^{29}$. The antiobesity effect of vitamin A supplementation might be, in part, due to its ability to decrease $11 \beta$-HSD1 activi$\mathrm{ty}^{49}$. MS seems to be associated with vitamin D deficien- cy and, accordingly, vitamin D status optimization seems to improve MS features ${ }^{50}$. A low-calcium diet alters GC metabolism leading to hepatic up regulation of $11 \beta$-HSD ${ }^{29}$; instead a high intake of calcium is associated with a low prevalence of $\mathrm{MS}^{51}$.

\section{OutLook}

The pathogenesis of the MS is multiple and still poorly understood. No single factor has yet been identified as an underlying causal factor; however, we strongly believe that visceral obesity might be the key feature in the pathogenesis of this syndrome. As a matter of fact, visceral obesity seems to be the major predictor of MS incidence ${ }^{52}$. In the context of stress response, GC direct effects are known for a long time ${ }^{12}$. Nevertheless the role of chronic stress and the hyperactivity of the HPA axis in the development of the MS remain not fully understood. The main putative mechanisms by which GC can promote visceral obesity are reviewed (Figure 2). Briefly, the release of GC induces lipolysis and the release of VLDL, increasing free fatty acids in circulation that are taken up for storage by other tissues, such as the visceral adipose tissue. GC promotes proliferation and differentiation of visceral adipose tissue via different mechanisms. For example, indirectly by enhancing the release of NYP, which is known by its action on adipocytes maturation, proliferation and angiogenic pathways. High levels of GC lead to an abnormal release of adipocytokines, such as leptin and adiponectin, which ultimately contributes to visceral fat accumulation. However, not all patients with MS present visceral obesity. As mentioned in a previous publication by our group, the size of abdominal adipocytes seems to be as important as the amount of accumulated fat in the abdominal cavity ${ }^{52}$. In fact, findings from our lab have shown that large adipocytes are more prone to rupture, and cell rupture constitutes a focus of inflammation that incites metabolic and immune responses leading to a series of pathogenic complications of obesity ${ }^{53}$. On the other hand, it is important to have in mind the 'cross-talk' between adipose tissue and other body systems, such as the immune system, since metabolic changes induced by GC release tend to be feed-forward and may, thus, lead to an endless spiral.

\section{Conclusion}

The major aim of this article was to describe literature evidences suggesting a causative role of GC in the development of MS. GCs are stress hormones with a wide spectrum of physiological effects and have been implicated in the pathophysiology of the MS. The pathophys- 


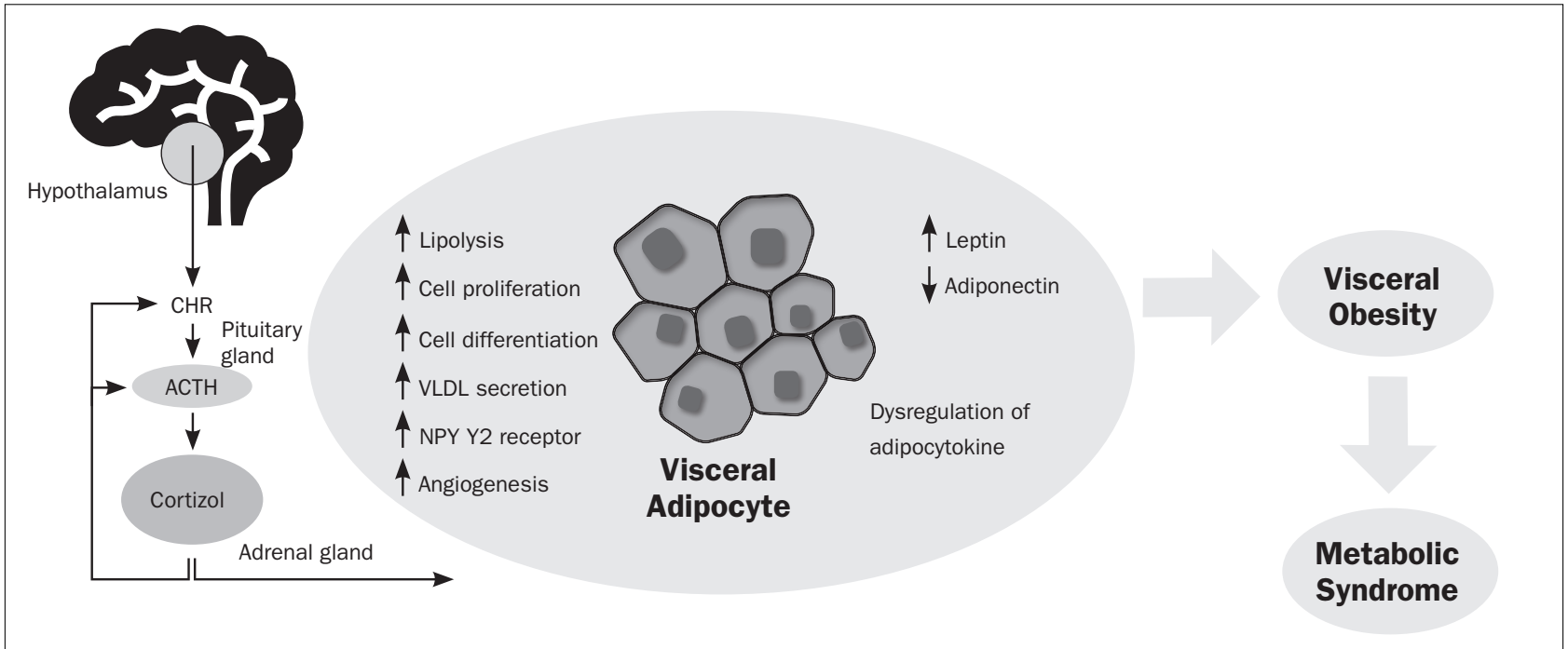

FIGURE 2 Putative mechanisms involved in GC release, visceral adipose tissue accumulation and the pathogenesis of Metabolic Syndrome. Cortisol, the major glucocorticoid in humans, is secreted in response to stressful events under the control of the hypothalamic- pituitary-adrenal axis. Cortisol exerts several cellular and metabolic effects in adipocytes, eventually promoting visceral obesity and the development of Metabolic Syndrome. CRH - corticotropin-releasing hormone; ACTH - adrenocorticotropic hormone.

iology of this syndrome remains partially understood, although available evidence hypothesized that hyperactivity of the HPA axis due to chronic stress and a state of functional hypercortisolism may have a central role in the pathogenesis of both abdominal obesity and insulin resistance. Data obtained from animal studies showed that exposure to both physical and psychological chronic stress was characterized by abnormal GC levels and several features of the MS. Although direct similar evidences are lacking in humans, epidemiological data have been providing evidence for a significant positive association between GC levels and the MS. In this review some of the underlying mechanisms supporting this hypothesis are highlighted. In our point of view, shared by others, one candidate that might be the link between chronic stress, GC and the MS, being probably the main trigger for the development of this syndrome, is visceral adiposity. Consequently, stress management programs, capable of effectively decrease circulatory GC levels, may constitute an important tool in patients at risk or suffering from MS.

\section{Acknowledgements}

The authors are grateful to Eng. Joana Afonso, who contributed with an original illustration for this paper (Figure 2).

\section{Resumo}

Cortisol: o vilão na Síndrome Metabólica?
Objetivo: Este artigo revê o estado da arte relativamente à associação entre as ações dos glicocorticoides e a obesidade e a resistência à insulina, dois dos principais componentes da síndrome metabólica.

Métodos: Uma revisão da literatura nas bases de dados PubMed e SciELO foi realizada usando os seguintes termos: estresse, síndrome metabólica, glicocorticoides, obesidade, resistência à insulina, eixo hipotálamo-hipófisesuprarrenal e 11ß-hidroxiesteróide desidrogenase.

Resultados: $\mathrm{O}$ estresse crônico, principalmente através da desregulação do eixo hipotálamo-hipófise-suprarrenal, promove a acumulação de gordura visceral. Reciprocamente, a obesidade promove um estado inflamatório sistêmico de baixo grau, mediado por alterações na secreção de adipocinas, que cronicamente podem estimular e perturbar o sistema de estresse. Esse círculo vicioso, provavelmente iniciado pela disfunção do tecido adiposo visceral, pode ser o mecanismo primário que conduz ao desenvolvimento da síndrome metabólica.

Conclusões: Um conhecimento mais aprofundado sobre os mecanismos envolvidos na associação entre a liberação de glicocorticoides, a obesidade e o diabete tipo 2 pode ser útil na prevenção e tratamento da síndrome metabólica.

Unitermos: estresse; glicocorticoides; obesidade; resistência à insulina; síndrome metabólica; $11 \beta$-hidroxiesteroide desidrogenase. 


\section{References}

1. Kyrou I, Tsigos C. Stress hormones: physiological stress and regulation of metabolism. Curr Opin Pharmacol. 2009;9:787-93.

2. Pasquali R, Vicennati V, Cacciari M, Pagotto U. The hypothalamic-pituitaryadrenal axis activity in obesity and the metabolic syndrome. Ann N Y Acad Sci. 2006;1083:111-28.

3. Kyrou I, Chrousos G, Tsigos C. Stress, visceral obesity, and metabolic complications. Ann N Y Acad Sci. 2006;1083:77-110.

4. McWwen BS. Stress, adaptation, and disease. Allostasis and allostatic load. Ann N Y Acad Sci. 1998;840:33-44

5. Iwasaki Y, Takayasu S, Nishiyama M, Tsugita M, Taguchi T, Asai M, et al. Is the metabolic syndrome an intracellular Cushing state? Effects of multiple humoral factors on the transcriptional activity of the hepatic glucocorticoidactivating enzyme (11beta-hydroxysteroid dehydrogenase type 1) gene. Mol Cell Endocrinol. 2008;285:10-8.

6. Flegal KM, Graubard BI, Williamson DF, Gail MH. Excess deaths associated with underweight, overweight, and obesity. JAMA. 2005;293:1861-7.

7. Shomali M. Diabetes treatment in 2025: can scientific advances keep pace with prevalence? Ther Adv Endocrinol Metab. 2012;3:163-73.

8. Brunner EJ, Hemingway H, Walker BR, Page M, Clarke P, Juneja M, et al. Adrenocortical, autonomic, and inflammatory causes of the metabolic syndrome: nested case-control study. Circulation. 2002;106:2659-65.

9. Vicennati V, Pasqui F, Cavazza C, Pagotto U, Pasquali R. Stress-related development of obesity and cortisol in women. Obesity. 2009;17:1678-83.

10. Chandola T, Brunner E, Marmot M. Chronic stress at work and the metabolic syndrome: prospective study. BMJ. 2006;332:521-5.

11. Rosmond R, Dallman MF, Björntorp P. Stress-related cortisol secretion in men: relationships with abdominal obesity and endocrine, metabolic and hemodynamic abnormalities. J Clin Endocrinol Metab. 1998;83:1853-9.

12. Charmandari E, Tsigos C, Chrousos G. Endocrinology of the stress response. Annu Rev Physiol. 2005;67:259-84.

13. Vegiopoulos A, Herzig S. Glucocorticoids, metabolism and metabolic diseases. Mol Cell Endocrinol. 2007;275:43-61.

14. Wang M. The role of glucocorticoid action in the pathophysiology of the Metabolic Syndrome. Nutr Metab (Lond). 2005;2:3.

15. Tsigos C, Chrousos GP. Hypothalamic-pituitary-adrenal axis, neuroendocrine factors and stress. J Psychosom Res. 2002;53:865-71.

16. Torpy DJ, Ho JT. Corticosteroid-binding globulin gene polymorphisms: clinical implications and links to idiopathic chronic fatigue disorders. Clin Endocrinol (Oxf). 2007;67:161-7

17. Anagnostis P, Athyros VG, Tziomalos K, Karagiannis A, Mikhailidis DP Clinical review: the pathogenetic role of cortisol in the metabolic syndrome: a hypothesis. J Clin Endocrinol Metab. 2009;94:2692-701.

18. Wamil M, Seckl JR. Inhibition of 11beta-hydroxysteroid dehydrogenase type 1 as a promising therapeutic target. Drug Discov Today. 2007;12:504-20.

19. Walker BR, Andrew R. Tissue production of cortisol by 11beta-hydroxysteroid dehydrogenase type 1 and metabolic disease. Ann N Y Acad Sci. 2006;1083:165-84.

20. Walker BR. Extra-adrenal regeneration of glucocorticoids by 11 betahydroxysteroid dehydrogenase type 1: physiological regulator and pharmacological target for energy partitioning. Proc Nutr Soc. 2007;66:1-8.

21. Rosmond R. Role of stress in the pathogenesis of the metabolic syndrome. Psychoneuroendocrinology. 2005;30:1-10.

22. Brown ES. Effects of glucocorticoids on mood, memory, and the hippocampus. Treatment and preventive therapy. Ann N Y Acad Sci. 2009; 1179:41-55.

23. Fiuza M, Cortez-Dias N, Martins S, Belo A. Síndrome metabólica em Portugal: Prevalência e implicações no risco cardiovascular: resultados do Estudo Valsim. Rev Port Cardiol. 2008;27:1495-529.

24. Shively CA, Register TC, Clarkson TB. Social stress, visceral obesity, and coronary artery atherosclerosis: product of a primate adaptation. Am J Primatol. 2009;71:742-51

25. Ribeiro Filho FF, Mariosa LS, Ferreira SR, Zanella MT. Gordura visceral e síndrome metabólica: mais que uma simples associação. Arq Bras Endocrinol Metab. 2006;50:230-8.

26. Haun D, Pitanga F, Lessa I. Razão cintura/estatura comparado a outros indicadores antropométricos de obesidade como preditor de risco coronariano elevado. Rev Assoc Med Bras. 2009;55:705-11.

27. Moller DE, Kaufman KD. Metabolic syndrome: a clinical and molecular perspective. Annu Rev Med. 2005;56:45-62.
28. Shively CA, Laber-Laird K, Anton RF. Behavior and physiology of social stress and depression in female cynomolgus monkeys. Biol Psychiatry. 1997;41:871-82.

29. Pereira CD, Azevedo I, Monteiro R, Martins MJ. 11ß-Hydroxysteroid dehydrogenase type 1: relevance of its modulation in the pathophysiology of obesity, the metabolic syndrome and type 2 diabetes mellitus. Diabetes Obes Metab. 2012;14:869-81.

30. Rask E, Walker B, Söderberg S, Livingstone DE, Eliasson M, Johnson O, et al. Tissue-specific changes in peripheral cortisol metabolism in obese women: increased adipose 11beta-hydroxysteroid dehydrogenase type 1 activity. J Clin Endocrinol Metab. 2002;87:3330-6.

31. Stewart PM, Boulton A, Kumar S, Clark PM, Shackleton CH. Cortisol metabolism in human obesity: impaired cortisone--> cortisol conversion in subjects with central adiposity. J Clin Endocrinol Metab. 1999;84:1022-7.

32. Rask E, Olsson T, Söderberg S, Andrew R, Livingstone DE, Johnson O, et al. Tissue-specific dysregulation of cortisol metabolism in human obesity. J Clin Endocrinol Metab. 2001;86:1418-21.

33. Wajchenberg BL. Subcutaneous and visceral adipose tissue: their relation to the metabolic syndrome. Endocr Rev. 2000;21:697-738.

34. Peckett AJ, Wright DC, Riddell MC. The effects of glucocorticoids on adipose tissue lipid metabolism. Metabolism. 2011;60:1500-10.

35. Espíndola-Antunes D, Kater CE. Adipose tissue expression of 11betahydroxysteroid dehydrogenase type 1 in Cushing's syndrome and in obesity. Arq Bras Endocrinol Metab. 2007;51:1397-403.

36. Christ-Crain M, Kola B, Lolli F, Fekete C, Seboek D, Wittmann G, et al. AMPactivated protein kinase mediates glucocorticoid induced metabolic changes: a novel mechanism in Cushing's syndrome. FASEB. J 2008;22:1672-83.

37. Walker BR. Cortisol - cause or cure for metabolic syndrome? Diabet Med. 2006;23:1281-8.

38. Joharapurkar A, Dhanesha N, Shah G, Kharul R, Jain M. 11ß-Hydroxysteroid dehydrogenase type 1: potential therapeutic target for metabolic syndrome. Pharmacol Rep. 2012;64:1055-65.

39. Costa J, Duarte J. Tecido adiposo e citocinas. Acta Med Port. 2006;19:251-6.

40. Dimitriadis G, Leighton B, Parry-Billings M, Sasson S, Young M, Krause U, et al. Effects of glucocorticoid excess on the sensitivity of glucose transport and metabolism to insulin in rat skeletal muscle. Biochem J. 1997;321:707-12.

41. Ikeoka D, Madjer J, Pieber T. Adipose tissue, inflammation and cardiovascular disease. Rev Assoc Med Bras. 2010;56:116-21.

42. Vasseur F, Meyre D, Froguel P. Adiponectin, type 2 diabetes and the metabolic syndrome: lessons from human genetic studies. Expert Rev Mol Med 2006;20:1-12.

43. Hermsdorff HH, Monteiro JB. Gordura visceral, subcutânea ou intramuscular: onde está o problema? Arq Bras Endocrinol Metab. 2004;48:803-11.

44. Nieuwenhuizen AG, Rutters F. The hypothalamic-pituitary-adrenal-axis in the regulation of energy balance. Physiol Behav. 2008;94:169-77.

45. Warne JP. Shaping the stress response: Interplay of palatable food choices, glucocorticoids, insulin and abdominal obesity. Mol Cell Endocrinol. 2009;300:137-46

46. Adam TC, Epel ES. Stress, eating and the reward system. Physiol Behav. 2007;91:449-58.

47. Seckl JR, Morton NM, Chapman KE, Walker BR. Glucocorticoids and 11betahydroxysteroid dehydrogenase in adipose tissue. Recent Prog Horm Res. 2004;59:359-93.

48. Wajchenberg BL, Nery M, Cunha MR, Silva ME. Adipose tissue at the crossroads in the development of the metabolic syndrome, inflammation and atherosclerosis. Arq Bras Endocrinol Metab. 2009;53:145-50.

49. Sakamuri VP, Ananthathmakula P, Veettil GN, Ayyalasomayajula V. Vitamin A decreases pre-receptor amplification of glucocorticoids in obesity: study on the effect of vitamin A on 11beta-hydroxysteroid dehydrogenase type 1 activity in liver and visceral fat of WNIN/Ob obese rats. Nutr J. 2011;10:70

50. Al-Daghri N, Alkharfy K, Al-Saleh Y, Al-Attas O, Alokail M, Al-Othman A, et al Modest reversal of metabolic syndrome manifestations with vitamin D status correction: a 12-month prospective study. Metabolism. 2012;61:661-6.

51. Liu S, Song Y, Ford ES, Manson JE, Buring JE, Ridker PM. Dietary calcium, vitamin $\mathrm{D}$, and the prevalence of metabolic syndrome in middle-aged and older U.S. women. Diabetes Care. 2005;28:2926-32.

52. Azevedo A, Santos A, Ribeiro L, Azevedo I. The metabolic syndrome. In: Soares R, Costa C, editors. Oxidative stress, inflammation and angiogenesis in the metabolic syndrome. Netherlands: Springer; 2009. p.1-19

53. Monteiro R, Castro P, Calhau C, Azevedo I. Adipocyte size and liability to cell death. Obes Surg. 2006;16:804-6. 